\title{
Pathosformel et mythe du progrès dans l'œuvre de Warburg
}

Silvia Ferretti

\section{(2) OpenEdition}

12 Journals

Édition électronique

URL : http://journals.openedition.org/imagesrevues/2834

DOI : 10.4000/imagesrevues.2834

ISSN : $1778-3801$

Éditeur :

Centre d'Histoire et Théorie des Arts, Groupe d'Anthropologie Historique de l'Occident Médiéval, Laboratoire d'Anthropologie Sociale, UMR 8210 Anthropologie et Histoire des Mondes Antiques

\section{Référence électronique}

Silvia Ferretti, «Pathosformel et mythe du progrès dans l'œuvre de Warburg », Images Re-vues [En ligne], Hors-série 4 | 2013, mis en ligne le 30 janvier 2013, consulté le 30 janvier 2021. URL : http:// journals.openedition.org/imagesrevues/2834 ; DOI : https://doi.org/10.4000/imagesrevues.2834

Ce document a été généré automatiquement le 30 janvier 2021.

Images Re-vues est mise à disposition selon les termes de la Licence Creative Commons Attribution Pas d'Utilisation Commerciale 4.0 International. 


\title{
Pathosformel et mythe du progrès dans l'œuvre de Warburg
}

\author{
Silvia Ferretti
}

1 Hermann Usener, dans son essai de 1904 intitulé Mythologie, qui a été vu comme une sorte de testament spirituel ${ }^{1}$, considérait Vico comme le précurseur des études modernes sur le mythe. Il évoquait aussi un autre Italien, Tito Vignoli, remarquant que celui-ci limitait le mythe à sa pure fonction psychique, la personnalisation d'un phénomène naturel ${ }^{2}$.

Usener avait écrit en 1881 une recension sur le livre de Vignoli Mito e scienza, qui venait alors d'être traduit en allemand, où il ironisait sur la prétention du philosophe

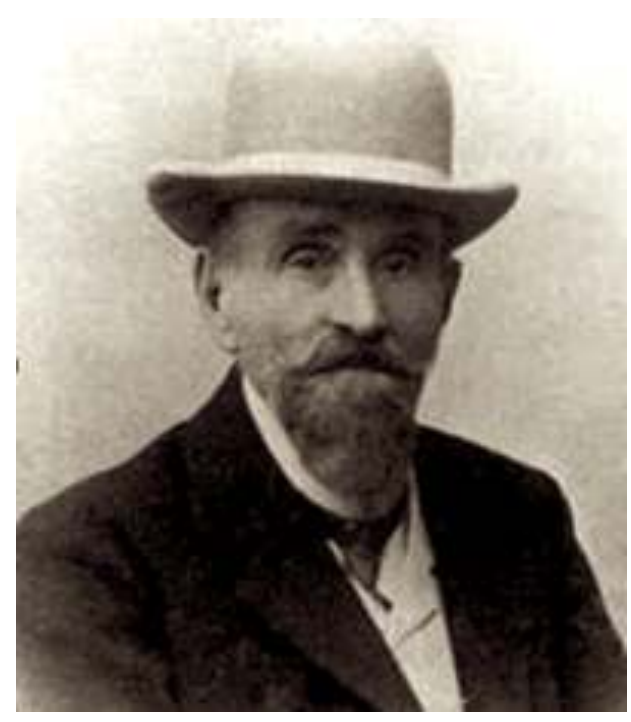
positiviste de se mesurer à Vico. Usener distinguait clairement l'apport fondamental de Vico à l'anthropologie, l'étude des peuples et des religions, un apport de tout autre mesure que la fort modeste contribution de Vignoli hypothéquée par une ingénuité de fond. Il lui reconnaissait cependant le mérite d'émettre quelques "étincelles" originales, qui seraient susceptibles d'allumer des "incendies" dans des domaines culturels différents ${ }^{3}$.

Usener s'occupait à cette époque de l'édition de son recueil monumental de fragments d'Epicure et commençait à concevoir les prémisses de son essai de mythologie comparée : les Götternamen ${ }^{4}$. Il ne se limita pas à lire avec curiosité le livre de Vignoli et à le traiter avec quelque sarcasme, car il en conseilla la lecture à un étudiant éclectique qui suivait ses cours à Bonn, Aby Warburg. L'ouvrage de Vignoli en allemand se trouve 
encore actuellement dans la Bibliothèque de l'Institut Warburg à Londres, avec des notes de sa main et même quelques passages soulignés de façon emphatique.

Cassirer lui-même, dans la Philosophie des formes symboliques, cite abondamment et favorablement l'ouvrage de Vignoli; il en choisit des passages caractéristiques de son environnement positiviste, les prend en considération avec la distanciation historique nécessaire et en tire - de façon tout à fait originale - ce qui peut lui être utile. Selon lui l'analyse positiviste de Vignoli est intéressante car elle décrit le "parallélisme de la formation des mythes" et soutient l'existence d'une règle de la naissance et du développement du mythe, une norme commune à toutes les cultures. L'atout de Vignoli, qui est aussi sa contradiction interne, est que quoique se déclarant empiriste, il n'en croit pas moins en un a priori de la fonction mythologique, une fonction innée dans l'esprit qui ne disparaît pas avec l'émergence de la science mais demeure à ses côtés ${ }^{5}$.

5 Cassirer revient sur Vignoli dans le troisième volume de son œuvre, à propos de la possibilité de retracer une origine biologique du mythe dans la psychologie animale, selon les canons de l'évolutionnisme. L'un des thèmes de recherche les plus innovants du positivisme était en effet celui de l'étude du monde perceptif des animaux, une recherche qui a eu d'excellentes répercussions dans le champ de la psychologie de la perception humaine au XXe siècle.

6 Cassirer ne dédaigne pas la multiplicité des lectures et des centres d'intérêt de Vignoli, de la littérature à la science et à la philosophie ; il sent une proximité dans cette façon d'enquêter. Il apprécie en particulier la tendance de Vignoli à donner une ligne gnoséologique à ses thèses, sur la base d'une psychologie de l'appréhension.

7 On peut imaginer que ce sont là les motifs qui des années auparavant avaient déjà suscité la curiosité de Warburg, suivant là son instinct d'ouverture à de nouvelles directions de recherche dans des territoires assez éloignés de son terrain en histoire de l'art (kunstwissenschaftlich). Warburg trouve dans Mito e scienza, le thème de l'unité fondamentale et de la collaboration entre diverses disciplines scientifiques, historiques, psychologiques, que lui-même posait à la base de ses études sur la Renaissance et qui l'a beaucoup rapproché de Cassirer dans les dernières années de sa vie.

8 La correspondance entre Warburg et Cassirer témoigne d'une compréhension réciproque où transparaît une haute affinité spirituelle et sensibilité. Il arrive dans ces lettres que Cassirer clarifie en deux mots sa pensée sur la forme symbolique en distinguant ses deux composantes, la Gestalt, une unité de fonction symbolique, et la Bildung, l'élément de la formation en processus ${ }^{6}$.

9 C'est là, - ainsi que dans le Tagebuch, ce journal où Warburg, Gertrud Bing et plus rarement Fritz Saxl, notent des idées, des problèmes, des découvertes du travail quotidien au sein de l'institut de Hambourg ${ }^{7}$-, que commence à prendre forme et à se déclarer l'intérêt de Warburg pour Giordano Bruno, dont la lecture lui avait été suggérée par Cassirer. Ce qui le frappe chez Giordano Bruno c'est cette harmonie entre les énergies cosmiques et la conscience rationnelle; il y retrouve les anciennes mythologies cosmologiques qu'il avait observées dans leur transmigration entre orient et occident ${ }^{8}$.

10 A l'époque où il lit Vignoli, Warburg lit aussi le texte de Darwin The Expression of Emotion in Animals and Men, qui revêt une grande importance pour sa conception de l'expression et du geste : il l'utilisera pour reformuler et amplifier une méthode visant à servir une 
science de l'art. La mentalité évolutionniste s'affirme avec force au XIXe siècle et il peut apparaître étrange à ceux qui connaissent le langage et la pensée de Warburg, de découvrir quelles pouvaient être ses sympathies avec ce milieu culturel. Nous savons en effet que l'orientation de sa pensée s'accorde plutôt sur celle des ennemis déclarés du positivisme, comme Burckhardt et Nietzsche, et que son regard aimait à se tenir à proximité de l'antiquité grecque qui, à travers les multiples coulisses de l'histoire, était revenu à la surface lors de la Renaissance, tout en s'accompagnant d'une volonté de confrontation et d'affirmation de soi typique des époques qui savent établir un rapport fort avec la tradition.

11 Warburg n'a pas le caractère conciliant de Cassirer qui tend souvent à résoudre avec optimisme les différends d'opinion avec ses contemporains en partant d'une vision de l'histoire des idées comme croissance progressive et dépassement des contradictions par un développement dialectiques, selon le schéma hégélien du devenir de l'esprit. Warburg n'a jamais suivi les visions universalistes et dogmatiques typiques de certains positivistes comme de nombreux idéalistes. Il se sert de théories philosophiques, historiographiques, anthropologiques et psychologiques pour retracer les motivations, cachées ou non, qui mènent les hommes d'une certaine époque à s'entourer de certains signes, symboles, styles et images, plutôt que d'autres.

Dans son œuvre, les problèmes et les solutions forment un réseau complexe de liens historiographiques qui ne sont ni linéaires, ni de causalité, ni abstraits ni généralisants, mais qui reposent sur une série d'épreuves de vérification, sur un vaste territoire interdisciplinaire de matériaux historiques soumis à la lumière de la remémoration. On reconnaît désormais combien était novatrice, dans son étude sur l'image artistique, son idée d'un rapport entre magie et sciences au début de l'époque moderne, son intuition d'une racine commune entre le mot et l'image dans l'expérience primaire de l'humanité, et combien une notion qu'il a forgée, la "formule de pathos"(Pathosformel), a contribué à amplifier la possibilité d'aborder plus intimement le message artistique.

Warburg célèbre la renaissance des divinités olympiques, détentrices de la forme parfaite (il dira plus tard "organique $»^{10}$ ), porteuses d'une extraordinaire vitalité emphatique suggérée par le mouvement des drapés et des chevelures mues par un souffle virtuel. Le renouveau de ces signes permet de renouer avec le sens apollinien et figuratif d'une dimension de la mesure, et de confirmer son propre rôle dans le temps historique. Ces figures de dieux, dotées de pouvoir de métamorphose, sont ensuite devenues des figures de saints et des scènes tirées de la religion judéo-chrétienne; à une certaine époque, elles ont été voilées, rendues prisonnières d'armures statiques et pesantes, maladroites si ce n'est pas monstrueuses, dans des structures qui communiquent de la signification mais pas le signe immédiat de la vitalité. Mais c'est ainsi qu'elles ont réussi à se conserver jusqu'au renouveau de la mobilité et de l'agitation de l'antiquité: le choix d'imiter le style des Anciens est considéré par Warburg comme une libération de la superstition et de l'obscurantisme.

Le progrès réside dans ces éclairs d'une antique intuition du monde dans son combat pour réussir à s'exprimer. Il faut alors « interpréter les figures de l'olympe païen non pas comme de simples phénomènes artistiques, mais comme des êtres religieux » pour comprendre pourquoi elles furent si difficiles à combattre de la part des cultures ennemies du paganisme ${ }^{11}$.

15 Selon Warburg, la forme-archétype de l'énergie prit origine dans une situation de peur, de risque et de souffrance qui trouva la voie de l'expression religieuse ou artistique. 
Cette forme de structuration se reproduit consciemment ou inconsciemment, chaque fois que les hommes tentent de recomposer, dans le cosmos immédiatement perceptible des images, une expérience qui se représente toujours, pour des raisons individuelles ou historiques, comme chaotique. C'est la raison pour laquelle il est important d'identifier "les symptômes d'un âge critique de transition", selon une expression qui se répète souvent chez Warburg ${ }^{12}$.

la douleur, de la peur ou de la pitié, pour conférer de la force à une image, répond aussi à l'exigence de rendre cette image plus durable, grâce à la force de l'impression qu'elle engendre chez le spectateur. Au milieu du XVIIIe siècle, Edmund Burke remarquait déjà que les impressions de l'âme qui durent le plus, et qui donc allongent la vitalité des fonctions cognitives et rendent plus active l'imagination, étaient liées aux passions de la souffrance et de la mort, à condition qu'elles se manifestent à une certaine distance du deuil ou de l'événement douloureux lui-même ${ }^{13}$.

17 En 1902 Warburg observait: "Quand des manières contradictoires de concevoir la vie jettent les membres isolés de la société dans des affrontements mortels et leurs inspirent une passion unilatérale, elles causent irrésistiblement le déclin de la société; et pourtant, ce sont en même temps des forces qui favorisent l'épanouissement de la civilisation la plus haute, quand à l'intérieur de l'individu, ces mêmes contradictions s'atténuent, s'équilibrent, et au lieu de se détruire, se fécondent mutuellement, apprenant ainsi à élargir l'ensemble de la personnalité. C'est sur ce terrain que croît la fleur de la civilisation de la Renaissance florentine ${ }^{14}$.

18 On sait que la Pathosformel implique une polarité de par sa formation même, puisqu'elle naît d'oppositions excessives dans l'âme, mais aussi en raison de sa transmigration temporelle et géographique: c'est une formule qui repose naturellement dans la mémoire de l'humanité et peut ressurgir à des époques, dans des zones géographiques et des cultures très éloignées et non communicantes ${ }^{15}$. Une telle voie d'accès à la recherche historique est bien sûr peu conforme à la mentalité positiviste et à la conception linéaire du temps historique qui se conçoit comme un progrès unidirectionnel vers des formes plus rationnelles de la société et de la connaissance. En réalité on y perçoit plutôt un écho de la théorie de Vico des "cours et recours", une théorie que Warburg pourrait avoir connue dans les leçons et les textes d'Usener et qu'il a certainement repérée chez Vignoli.

19 Warburg n'a cependant pas en tête la formulation d'une théorie univoque sur l'histoire: il examine tout concours de circonstance possible lui permettant de tracer un itinéraire mental à travers des temps, lieux différents, des situations diversifiées. Vignoli savait que Vico n'était en rien un théoricien du progrès historique. Dans un livre de 1864, Di una teoria razionale del progresso, ouvrage imprégné de darwinisme et d'analyses physicocosmologiques appelées en soutien à l'idée de progrès, Vignoli affirme son admiration pour le génie de Vico, gloire de la philosophie italienne, mais il ne soutient toutefois pas le "cercle perpétuel et stérile" dans lequel le philosophe napolitain enclot l'humanité. De fait, à l'époque du positivisme, les principaux interprètes de Vico s'accorderont toujours contre la théorie des "cours et recours" ${ }^{16}$. Mais vu qu'il l'avait inséré dans sa "théorie du progrès", Vignoli devait admirer chez Vico quelque chose de plus vaste pouvant intéresser un amateur aussi éclectique que lui.

20 C'était sans doute le cas de la psychologie poétique développée par Vico, qui depuis est devenue fondamentale pour les sciences historiques, mais il s'agissait aussi de l'analyse 
que proposait Vico quant à l'intelligence primitive comme origine du mythe et du symbole. Quoique Vico ait, en tant que "platonicien", une vision "mythologique" de l'histoire, Vignoli montre un grand intérêt pour un aspect de sa pensée qui deviendra peu après de grande importance pour les sciences de la culture. Cet aspect concerne l'esthétique ou plus exactement le langage: le développement, avec Vico, de la philologie comparée, une discipline qui s'était affirmée en Europe et que Vignoli suivait avec un grand intérêt ${ }^{17}$.

Cette attention spécifique de Vignoli, qui se manifeste avec plus d'ampleur dans Mito $e$ scienza, contribua pour beaucoup à l'établissement du lien avec l'école philologique de Bonn où le jeune Warburg écoutait Usener.

Par ailleurs on trouve chez Vignoli, au-delà d'exaltations convenues sur l'évolutionnisme historique, des thèses qui purent être partagées par Usener, Warburg et plus tard Cassirer. Le thème de la psychologie comparée, qui étudie la continuité des représentations entre les animaux et les hommes, mène Vignoli sur les traces d'un phénomène considéré comme originel dans toutes les cultures: la personnification de l'émotion soudaine. Les événements naturels et les apparitions sensibles transformées en symboles sont à l'origine des superstitions, des rituels magiques, des religions et des mythologies, et président aussi à la naissance primordiale de la recherche scientifique $^{18}$. Dans la philosophie des formes symboliques de Cassirer, le passage de l'individualisation à la détermination des espèces joue un rôle de premier rang, de même que le passage du fait particulier et personnel au type qui est à la base de la formation symbolique la plus évoluée. Dans Mito e scienza, Vignoli pense à une sorte d'acte unique de conscience dont découlent aussi bien le mythe que la science, comme deux courants parallèles qui cependant à un certain moment de l'histoire se surpassent l'un l'autre, viennent à confluer pour se séparer de nouveau ${ }^{19}$. Il s'agit là d'une thèse confuse et faiblement théorisée qui révèle la contradiction intime au sein d'une conception évolutionniste qui doit élaborer un permanent retour à la surface de motifs ancestraux (on note d'ailleurs que même dans les grands textes du positivisme européen le mythe n'est jamais complètement réabsorbé dans la science).

On peut comprendre les difficultés auxquelles se heurte Vignoli dans sa tentative de sauver tout à la fois la continuité naturelle et le progrès de la civilisation qui comporte la mutation, le changement de direction, l'émergence d'éléments nouveaux. La succession qui va du mythe à la logique coexiste avec "l'évolution des notions abstraites", sans que l'auteur ne s'en rende compte. Ce sont là des problèmes que Cassirer se retrouvera à affronter, certes avec une conscience théorique et historiographique bien supérieure, pour donner alors des assises fonctionnelles à la forme symbolique et en assurer la variabilité.

Warburg trouve chez Vignoli cette confluence complexe de suggestions variées qui formera le motif central de sa recherche interdisciplinaire. Il n'y a aucune raison que la "religion du progrès" ne le trouble particulièrement car il vivait à un moment de son siècle où le positivisme était déjà entré en crise et il est probable qu'il percevait à sa façon les contradictions de la pensée de Vignoli comme une tentative de sortir du point de vue traditionnel sur le rapport mythe-science.

Par ailleurs il s'intéressait particulièrement à la question du statut mental de l'homme primitif, conçu chez Vignoli comme preuve de la progression de l'humanité vers des stades plus évolués pour aboutir à la civilisation de la science : c'est une question qui devient plus complexe chez Warburg, en particulier avec le problème du rapport entre 
les primitifs et l'antiquité en raison de sa recherche d'une anthropologie pouvant rendre compte aussi bien des conceptions de l'existence des primitifs que des conceptions gréco-antiques et modernes (que l'on pense au parallèle qu'il établit entre Athènes et Oraibi dans Schlangenritual, sur le 'rituel du serpent ${ }^{{ }^{20}}$ ). Selon une conception largement répandue dans la psychologie et l'anthropologie de son époque, Warburg considère que l'élément primitif, loin d'être rendu caduc par la civilisation et le savoir scientifique, demeure fondamental dans les comportements civilisés et revient à la surface à certains moments de l'histoire, dans les formes de choix expressifs de l'art et de la communication, ou bien sous la forme tragique de la folie collective, de la déflagration des impulsions destructives.

J. J. Bachofen appartient à ce groupe des théoriciens d'un scepticisme historique à contre courant à l'instar de Nietzsche et Burckhardt (les grands "inactuels"): ce spécialiste du matriarcat et de la religiosité chthonienne a contribué à renverser le rythme de l'évolution et la continuité de l'histoire culturelle. En accord avec Bachofen, Warburg ne considère pas les données historiques comme de simples faits, ni la recherche de sources comme le résultat de simples associations; il n'accepte pas le principe de l'association d'éléments psychiques proposé par le positivisme, une conception d'ailleurs sérieusement contestée si ce n'est démontée à son époque, par Bergson par exemple. L'interprétation des faits et des contextes consiste plutôt en un travail de décomposition et recomposition, il s'agit de percevoir et de suivre des empreintes grâce à l'intuition propre à l'historien, à sa conception des notions de temps et d'événement qui le mèneront à repérer sous des détails apparemment insignifiants, des expressions d'un dynamisme formant un style. Et pour Warburg le style ne montre jamais une évolution organique dans l'histoire de l'art ${ }^{21}$.

Je ne crois pas que Warburg ait jamais adhéré, en tant qu'homme du XIXe siècle, au positivisme ni surtout aux doctrines de l'évolution ${ }^{22}$, contrairement à ce que certains pensent pouvoir constater dans des théories comme la translation d'images et la conception héréditaire des instincts gestuels et des réactions spontanées et inconscientes. Le fait qu'il y ait selon lui, comme selon Freud, une espèce de réservoir inconscient de faits qui peuvent revenir se manifester sous des formes pathologiques ou non dans la conscience, n'a rien à voir avec le progrès ou l'évolution de l'humanité. Il semble plutôt que Warburg et Freud, sous des modalités différentes bien sûr, relèvent les manifestations d'une "malaise dans la civilisation", un dur effort d'adaptation aux nécessités imposées par le progrès scientifique et par les conditions économiques, l'accomplissement d'un travail qui réprime le sens spontané et naturel de la vie ou qui débouche sur l'expression de la génialité dans les œuvres artistiques.

Rappelons ici ce qu'avait dit Usener : "l'idée de progrès est associée à des étincelles de pensée" ${ }^{23}$ qui peuvent mener à des nombreuses découvertes et intuitions dans des domaines très variés et qui ont construit la modernité, comme l'idée de la peur de l'homme primitif face à l'inconnu et l'inexplicable, ou comme le passage du culte à la culture, de la foi dans la transcendance au mythe de la civilisation. J'entends par là que la foi dans la civilisation et dans un parcours rectiligne qui devrait nous y conduire a aussi produit (et contenait en soi, comme préexistant) un fort désir de retour aux sources "primitives", de curiosité pour les ambiguïtés du fétichisme; de même que la sécularisation a mené à un véritable approfondissement sur les origines du sacré et sur la religiosité, à la naissance de l'histoire comparée des religions, sans parler des études d'anthropologie et de l'extraordinaire diversification du phénomène de l'art au XXe 
siècle. Comme le dit Alain Michaud: Warburg reprend les clichés progressistes du positivisme pour les renverser ${ }^{24}$. Progrès et régression forment selon lui un couple qui procède en parallèle.

Contrairement à ce qui il a souvent été dit $^{25}$, la sagesse, selon Warburg, ne se perd pas dans la "banalité de la technique" ; elle se perd en raison de l'irruption de la folie dans l'individu comme dans la collectivité, et la folie peut être générée tant par les monstres de la magie et du mythe que par la violence de la technique. Warburg était très intéressé par le merveilleux dans le progrès technologique, il croyait à l'importance de la photographie pour la reproduction et l'étude des détails dans les œuvres d'art - et ceci bien avant Benjamin - mais aussi comme document de science de l'art et moyen de transmission des signes de la psychologie de son époque, comme le démontre la composition de l'Atlas.

Je ne pense pas que Warburg fasse partie des dénigreurs de la technologie entre le XIXe et le XXe siècle. La perception du péril qui dérive du progrès technologique, manifestée de façon explicite à la fin de la conférence sur le 'Rituel du serpent ${ }^{26}$, n'a rien à voir avec une pathologie de Warburg comme individu, mais renvoie plutôt à une pathologie du temps historique, comme une souffrance qui se répète à différents moments de l'histoire face à l'accélération des moyens et des rapports entre l'homme et un monde extérieur insoutenable, sans commune mesure avec le Denkraum, cet espace de la pensée qui nous assure une qualité de distanciation entre nous et le monde qui nous entoure.

Dans un fragment de 1891 Warburg dit: « Le jugement (Urteilskraft) est le produit du fait même de la distance entre sujet et objet; quand cette distance se fait valoir comme sentiment de distance dans le sujet $\gg{ }^{27}$.

L'essai de 1912 sur Sassetti et la Renaissance florentine se situe déjà dans cet ordre d'idées. Les sensations que cet ouvrage nous communique, en nous montrant la mutation de l'humeur de Sassetti dans son testament et dans ses choix d'emblèmes et de symboles de son pouvoir, interpellent ce qu'on pourrait appeler "le Devenir dans le Trépas". Le poète - ou le héros tragique - qui perçoit le présent comme avènement d'un monde différent, alors que tous autour de lui continuent à rester avec l'esprit tourné vers le passé, risque la mort, selon Hölderlin ${ }^{28}$. «Dans l'usage métaphorique des figures de l'antiquité, Sassetti aussi bien que Rucellai révèlent combien dans cette époque de transition de l'attitude subjective, ils aspiraient à un équilibre nouveau entre des énergies différentes" ${ }^{29}$.

Max Weber reconnut immédiatement en lisant "Les dernières volontés de Francesco Sassetti" que l'ami de Laurent de Médicis et de Poliziano était un personnage digne de ses recherches sur l'esprit du capitalisme. Weber écrivit à Warburg que le Lebensstil (style de vie) est toujours en contraste avec la Wirtschaftsform (le régime de l'économie) et que Sassetti se heurte à « une impossibilité de fonder un nouveau style de vie à partir des nécessités du pouvoir économique $»^{30}$.

34 Dans les "échanges de civilisation artistique", mythe et logos se superposent et s'alternent selon les vicissitudes de l'histoire. Le logos semble ainsi correspondre à la recherche continuelle et à la redécouverte cyclique des traces évidentes du mythe le long des voies explicites ou souterraines au cours de l'histoire. Warburg ne cherche pas des connexions ou des parentèles à partir d'un point de vue téléologique a priori et abstrait, ou selon un mécanisme d'enchaînement de causes à effets. Il révèle au 
contraire la prudence de l'historien empirique qui, comme l'archéologue, doit dégager de la boue et des déchets un nouvel objet avant de pouvoir dire de quoi il s'agit.

La structure même de la Pathosformel, sa consistance matérielle sous forme d'image visuelle, sa puissance presque tactile, mais aussi sa nature de schéma où ce qui est complexe et contradictoire se manifeste en une unité, comme s'il s'agissait de quelque chose de simple et d'immédiat; tout cela ne saurait s'accorder avec l'idée de progrès. Une telle formation sensible acquiert un caractère de solidité en tant que structure permanente mais elle est aussi protéiforme; elle ne répond pas à un goût constant mais oscille entre les pôles opposés de l'histoire et ne se manifeste que rarement en un tout organique: dans l'œuvre d'art. Lors de cette lutte entre les pôles de la contradiction, elle peut se dissimuler comme forme incomplète lorsque l'un des pôles triomphe temporairement sur l'autre, elle se conserve alors intacte mais voilée, jusqu'à ce qu'un choix de style bien précis ne l'amène à une nouvelle extériorisation.

Le mythe du progrès est beaucoup plus ancien que la foi dans la science du positivisme, et dès l'antiquité on assiste à une alternance dans les visions du monde entre mythe du progrès et mythe de la décadence. Il a été récupéré dans des idéologies politiques de toutes tendances et toutes l'ont aussi abandonné. Il est difficile de le théoriser au-delà d'un certain point car il n'existe pas de preuves certaines de sa consistance réelle. On peut dire seulement que, pour reprendre les mots de Benjamin, « du paradis souffle une tempête... ». La bataille de Warburg pour le droit des êtres humains à leur Denkraum, à une raisonnable possibilité de mettre sa propre existence à l'abri du hasard ou de la folie destructive de soi et des autres, cette lutte peut paraitre parfois comme une bataille pour le progrès, mais la conscience de l'exiguïté des probabilités qu'a la raison de l'emporter sur l'irrationnel, amène la pensée laïque, non religieuse, à constater l'impuissance des garanties de son salut.

\section{NOTES}

1. M. M. Sassi, "Dalla scienza delle religioni di Usener a Aby Warburg", in Aspetti di Hermann Usener filologo della religione, Pisa 1982, 80.

2. Sur Usener et Vignoli, ibid. 83 ss.

3. H. Usener, compte-rendu à Tito Vignoli, Mythos und Wissenschaft, in Deutsche Litteraturzeitung, Berlin, 2. Juli 1881, 1066-1067. L'œuvre de Vignoli a été publiée à Milan en 1879.

4. H. Usener, Götternamen. Versuch einer Lehre von der religiösen Begriffsbildung (1896). Rappellons que Psyche de Erwin Rodhe est paru en 1893.

5. Ernst Cassirer, Philosophie des formes symboliques, trad. J. Lacoste, Paris 1972, II, La pensée mythique, 37-38, et aussi III, La phénoménologie de la connaissance, trad. C. Fronty, 92-94.

6. Aby Warburg - Ernst Cassirer, Il mondo di ieri. Lettere, édité par Maurizio Ghelardi, Torino 2003, 54-55. S.Ferretti, Il demone della memoria, Casale Monferrato 1984 , G. Didi-Huberman, L'image survivante. Histoire de l'art et temps de fantômes selon Aby Warburg, Paris 2002, 434-435.

7. Aby Warburg, Tagebuch der kunstwissenschaftlichen Bibliothek Warburg, hrsg. von K. Michels und Ch. Schoell-Glass, Berlin 2001. 
8. A. Warburg, «Art Italien et Astrologie Internationale au Palazzo Schifanoia à Ferrara ", in Essais florentins, trad. S. Muller, Paris 1990.

9. A. Warburg, «L'art du portrait et la bourgeoisie florentine. Domenico Ghirlandajo à Santa Trinita. Les Portraits de Laurent de Médicis et de son entourage» (1902) , in Essais florentins cit. , 110. «Francesco Sassetti représente ce type de bourgeois intègre et intelligent d'une époque de transition qui, sans pose héroïque, rend justice au nouveau sans pour autant renoncer à l'ancien ».

10. Voir Aby Warburg, Atlas. Mnémosyne, introduction de Aby Warburg, où il parle de «style organique $»$.

11. A. Warburg, «La divination païenne et antique dans les écrits et les images à l'époque de Luther » (1920), in Essais florentins, cit. 250.

12. Ibid.

13. E. Burke, Enquiry on the Origine of our Ideas of Beautiful and Sublime (1757).

14. A. Warburg, « L'art du portrait... » op. cit, 110.

15. Voir S. Ferretti, "La polarità nella formazione filosofica e culturale di Aby Warburg", in Claudia Cieri Via, Pietro Montani, Lo sguardo di Giano, Torino 2004.

16. Benedetto Croce, Bibliografia Vichiana, II, Napoli 1948, 647-648. B. Croce, La filosofia di Giambattista Vico (1911).

17. Eugenio Garin, "Il positivismo italiano alla fine del secolo XIX fra metodo e concezione del mondo", in Giornale critico della filosofia italiana, LIX, 1980, 1-27.

18. T. Vignoli, Mito e scienza, op. cit., 76.

19. Ibid. , 97.

20. A.Warburg, Le Rituel du serpent. Récit d'un voyage en pays pueblo, Paris 2003.

21. A. Warburg, «Les dernières volontés de Francesco Sassetti » (1907) , in Essais florentins, cit., 169 ss..

22. Ernst Gombrich, "Warburg und der Evolutionismus des 19. Jahrhunderts", in Ekstatische Nymphe...trauernder Flußgott. Porträt eines Gelehrnten, Hrsg. R.Gallitz und B.Reimers, Hamburg 1995, 52 ss.

23. Voir Roland Kany, Mnemosyne als Programm, Tübingen 1987, 74.

24. Ph.A. Michaud, Aby Warburg et l'image en mouvement, Paris 1998, 33.

25. Kany , cit. 175.

26. Rituel du serpent, cit.

27. Fragment 189 cit. en M. Rampley, The Remembrance of Things Past. On Aby Warburg and W. Benjamin, Wiesbaden 2000, 43.

28. Fr.Hölderlin, Oeuvres I, Gallimard 1967, 656.

29. Warburg, Sassetti , op. cit. 195.

30. Max Weber, Werke GA, Tübingen 1992, II, 5 Briefe 1906-1908, 390-91. Sur Warburg lecteur de Weber M. Diers, Warburg aus Briefen, Weinheim 1991, 94.

\section{INDEX}

Mots-clés : Usener, Vignoli, Vico, Cassirer, forme symbolique, Denkraum, mythologie, science, philosophie 


\section{AUTEUR}

\section{SILVIA FERRETTI}

Professeur d'Esthétique à l'Université de Macerata, elle est l'auteur de Il demone della memoria. Simbolo e tempo storico in Warburg, Cassirer, Panofsky, Genova 1984, Antichi e moderni. L'elaborazione del passato, Pisa 2005, Apologia del gusto (avec Filippo Focosi), 2012. 